\title{
Calidad del servicio público de aseo urbano. Caso: Municipio Maracaibo
}

\author{
Sáez Alejandrina* \\ Machado Emily** \\ Godoy Enemarsy***
}

\section{Resumen}

En Venezuela el aseo urbano es considerado un servicio básico, cuya responsabilidad recae sobre los gobiernos locales. En tal sentido por ser catalogado básico, las fallas en la prestación del servicio genera manifestaciones de malestar de la población, como es el caso de la ciudad de Maracaibo, comportamiento que ha experimentado un auge, tanto por las graves consecuencias en la salubridad, como la incomodidad estética y el impacto ambiental ocasionados. Esto ha hecho pertinente, determinar la calidad del servicio público de aseo urbano domiciliario en el municipio Maracaibo, parroquias Francisco Eugenio Bustamante y Antonio Borjas Romero, constituyendo el objetivo principal del presente artículo. El diseño de investigación fue no experimental de campo, con un tipo de estudio descriptivo, para lo cual se elaboró y aplicó un instrumento de medición de la calidad con base en la percepción del usuario, siguiendo un muestreo por etapas. Finalmente, se obtuvo una medición de la calidad de servicio valorada en 2.228 , en una escala de 1.0 a 5.0, siendo 5.0 la máxima puntuación. En consecuencia se concluye que el nivel de calidad de servicio en las parroquias estudiadas es bajo.

Palabras clave: Calidad de servicio, servicio público, aseo urbano domiciliario.

Recibido: 07-12-10. Aceptado: 26-10-11

* Ingeniera Industrial (LUZ). Magister en Gerencia de Empresas: Mención Mercadeo (LUZ). Profesor Titular de la Facultad de Ingeniería, Universidad del Zulia. Teléfono: 0261-4128786. e-mail: asaez@fing.luz.edu.ve.

** Ingeniera Industrial (LUZ). Magister Scientiarium en Gerencia de Empresas (URBE). Profesor Agregado de la Facultad de Ingeniería, Universidad del Zulia. e-mail: emachado@fing.luz.edu.ve

*** Estudiante de Ingeniería Industrial de la Universidad del Zulia. e-mail: enemarsy.godoy@gmail.com 


\title{
Public Service Quality for Urban Sanitation. Case: The Maracaibo Municipality
}

\begin{abstract}
In Venezuela, urban sanitation is considered a basic service, for which local governments are responsible. Since it is catalogued as basic, failures in rendering this service generate manifestations of malaise in the population, as is the case with the city of Maracaibo, behavior that is on the increase due to serious health consequences as well as the aesthetic discomfort and environmental impact caused. Therefore, main objective of this article is to determine public service quality for urban household sanitation in the Maracaibo Municipality, Francisco Eugenio Bustamante and Antonio Borjas Romero Parishes. The research was a descriptive study with a non-experimental, field design, for which a quality measuring instrument based on user perception was elaborated and applied, according to a multistage sampling. Finally, a measurement for service quality valued at 2.228 was obtained, on a scale of 1.0 to 5.0 , with 5.0 being the highest score. Conclusions are that the service quality level in the parishes under study is low.
\end{abstract}

Key words: Service quality, public service, household urban sanitation.

\section{Introducción}

En Venezuela, la calidad de vida de los ciudadanos, así como el desarrollo urbano están siendo sensiblemente afectados por el impacto que en materia de eficiencia, efectividad y calidad tienen los servicios públicos en el desarrollo de una nación. El aseo urbano es considerado un servicio básico, que en Venezuela es responsabilidad del Gobierno Nacional y de los gobiernos locales. Existen lineamientos definidos en la Constitución Nacional, así como, legislaciones y ordenanzas municipales que determinan la logística para la prestación de este importante servicio. En el Municipio Maracaibo el organismo encargado de esta función es el Instituto Municipal de Aseo Urbano (IMAU).

El gobierno nacional ha venido impulsando cambios en el sistema político hacia un modelo con mayor responsabilidad social, sin embargo, las manifestaciones de malestar de la población, por las fa- llas en la recolección de los desechos domiciliarios, han experimentado un auge entre los ciudadanos, tanto por las graves consecuencias en la salubridad como la incomodidad estética y el impacto ambiental ocasionados. Todo esto, ha hecho pertinente determinar la calidad del servicio de aseo urbano domiciliario en la ciudad.

Dado la complejidad del servicio y el alcance de la investigación, el estudio se realizó por fases, siendo un primer paso hacia la consecución de este logro, elaborar una propuesta para la evaluación de la calidad del servicio según la percepción del usuario.

Es oportuno resaltar que esta propuesta fue sólo una aproximación inicial al diseño de un instrumento de medición de la calidad de servicio del aseo público urbano domiciliario, la cual, está enmarcada en un programa de investigación que busca profundizar en la determinación de las variables que inciden en la medición de la calidad de este servicio, 
Calidad del Servicio Público de Aseo Urbano: Caso: Municipio Maracaibo

Sáez Alejandrina; Machado Emily y Godoy Enemarsy

esto último constituye el objeto de este trabajo.

La investigación utilizó el modelo SERVPERF (calidad de servicio percibida), instrumento creado por Cronin y Taylor (1992 y 1994), como base para medir la calidad de servicio del aseo urbano desde la percepción del usuario. Es importante enfatizar el hecho que este es un modelo "genérico", es decir, puede aplicarse, en teoría, a cualquier tipo de servicio. No obstante, la adaptación del mismo para la evaluación de un servicio público tan importante en una urbe como la ciudad de Maracaibo, debe realizarse con mayor cuidado que para cualquier otro tipo de servicio, considerando por supuesto, la complejidad de la logística al momento de la prestación del mismo.

El instrumento utiliza la escala Likert, asociando a cada respuesta una puntuación, con la intención de transformar una apreciación cualitativa del ciudadano en una cuantitativa que permitiera medir la calidad de servicio del aseo urbano. Las opciones con que el usuario cuenta para responder varían desde estar fuertemente en desacuerdo, muy en desacuerdo, incluyendo una posición neutral ni en desacuerdo ni de acuerdo, hasta el otro extremo donde el entrevistado puede expresar estar muy de acuerdo o fuertemente de acuerdo con las declaraciones planteadas. A estas alternativas de respuesta se les asigna puntuaciones del 1 al 5 respectivamente.

En cuanto a la población objeto de estudio, ésta queda conformada por todos los domicilios (hogares) pertenecientes al municipio Maracaibo, generadores de desechos sólidos. Es pertinente resaltar que se delimitó el alcance de la investi- gación a dos parroquias: Francisco Eugenio Bustamante (FEB) y Antonio Borjas Romero (ABR), contando con un total de 59.503 hogares para el año 2010 , según estimaciones propias realizadas a partir de las proyecciones para el 2005 del Diario La Verdad. La unidad por medio de la cual se obtuvo la información fue el representante de cada hogar.

Una vez definida la población y conociendo que esta es finita pero muy grande, se diseñó un muestreo en dos etapas para garantizar una selección aleatoria de los elementos muestrales. En una primera etapa se utilizó un muestreo estratificado, considerando como estratos las parroquias objeto de estudio, logrando así garantizar que la muestra incluya sectores aleatoriamente seleccionados que pertenezcan a ambas parroquias de acuerdo a la proporción de hogares de cada una de ellas; mientras que en la segunda etapa se utilizó un muestreo por conglomerados, que contienen colecciones de elementos muestrales, para este caso, hogares o domicilios generadores de desechos sólidos. Los conglomerados se definieron como las manzanas dentro de cada sector, estimando el número de conglomerados a incluir en la muestra con base al promedio del número de domicilios dentro de cada manzana y la disponibilidad de recursos.

Para lograr establecer la cantidad de hogares a incluir en cada estrato, fue necesario conocer el porcentaje de usuarios que califican el servicio de aseo urbano con alta calidad, dicha proporción fue $3.6 \%$ y se estimó a partir de una muestra piloto de 83 representantes de hogares. 
Adicionalmente, con la información obtenida a partir de la prueba piloto se logró determinar la confiabilidad del instrumento utilizando el método Alfa Cronbach. El resultado de este índice fue de 0.869 , el cual se considera adecuado en función de que este coeficiente es aceptable si tiene valores comprendidos entre 0.75 y 0.90 .

Finalmente, con base en la disponibilidad de recursos se estableció el tamaño de la muestra en 314 representantes de hogares, a quienes se les aplicó el instrumento con la finalidad de medir la percepción que tienen de la calidad del servicio público de aseo urbano domiciliario.

\section{El servicio de aseo urbano}

El artículo 5 del Proyecto de Ley Orgánica de los Servicios Públicos (Asamblea Nacional, 2006), considera como servicios públicos domiciliarios los que presta el estado a los ciudadanos y ciudadanas directamente en sus viviendas, y en prioridad los servicios de electricidad, agua potable y, saneamiento y aseo domiciliario. Vinculado al concepto de servicio público, el servicio de aseo urbano domiciliario es de tipo I, desde la óptica del tipo de necesidad que satisface. Además, según Acurio et al. (1998) comprende las actividades relacionadas al manejo de los residuos sólidos municipales las cuales incluyen: almacenamiento, presentación para su recolección, recolección, transporte y transferencia, tratamientos, recuperación, reciclaje, y disposición final, siendo esta última imprescindible para el manejo. También abarca otras actividades tales como el barrido y limpieza de vías y áreas públicas, com- prende otros servicios especiales como la recolección de animales muertos.

Partiendo de lo establecido en la legislación venezolana, en la Ley Orgánica del Poder Público Municipal (2005), de acuerdo a lo señalado en el artículo 56 numeral 2, es competencia propia de los municipios la dotación y prestación de los servicios públicos incluyendo en éstos el aseo urbano y domiciliario.

De la revisión legislativa venezolana, en materia de servicios públicos y aseo urbano, se deriva entonces que los protagonistas de la prestación de este servicio son: el Alcalde de la ciudad, en su rol de primera autoridad y representante del poder municipal; el ciudadano, quien genera los desechos, y que debe tener una actitud proactiva en cuanto a qué hacer con la basura generada por él ; el sector productivo que genera una serie de bienes e insumos, que una vez en el mercado y desechados por el consumidor, forman parte de lo que denominados residuos sólidos y por supuesto, la empresa o institución encargada de proveer el servicio de aseo urbano domiciliario, sobre quien la alcaldía delega la prestación de este servicio público.

\section{Calidad de servicio y sus dimensiones}

Es importante destacar que la calidad es determinante en la percepción del cliente sobre un servicio. Pride et al (1997) definen la calidad de servicio "como la percepción que tienen los clientes sobre que también un servicio satisface o excede sus expectativas". En este sentido, Stanton (2004) señala que existen dos atributos de la calidad de servicio, el primero es que la calidad de servicio la 
Calidad del Servicio Público de Aseo Urbano: Caso: Municipio Maracaibo Sáez Alejandrina; Machado Emily y Godoy Enemarsy

define el cliente no el productor-vendedor, y la segunda, es que los clientes evalúan la calidad del servicio comparando sus expectativas con sus percepciones de cómo se efectúa este. Considerando entonces que la calidad en la prestación del servicio la establecen los usuarios, toda empresa debe medir con regularidad la satisfacción de los mismos, esto es, medir las percepciones de los clientes acerca de la calidad con la que una organización entrega u ofrece un servicio. En función de lo anteriormente expuesto, para la presente investigación se define calidad de servicio como el grado en que la oferta de un intangible satisface las expectativas del cliente o usuario.

Partiendo de la reflexión anterior, la cual supone que es el usuario quien valoriza el servicio que recibe, el punto de partida en la elaboración de cualquier instrumento para medir la calidad de un servicio consiste precisamente en definir cuáles son los factores o criterios que los usuarios consideran determinantes para evaluar la calidad del mismo. Zeithaml et al (1992), lograron identificar algunos de los elementos básicos de la calidad de servicio, destacando lo difícil que es medir la forma en que los clientes perciben la calidad, así como la necesidad de adaptar las dimensiones y medidas al contexto de investigación.

La conceptualización de Zeithaml et al (1992), sobre la calidad de servicios (modelo americano), supone que los clientes comparan sus expectativas con sus percepciones (lo que en términos de servicio reciben realmente) para cada una de las dimensiones clave en la experiencia del servicio, agrupándolas en los cinco elementos de calidad siguientes: confiabilidad, elementos tangibles, empatía, seguridad y capacidad de respuesta, descritas en el Cuadro 1.

En estas dimensiones se consideran los factores que el cliente toma en cuenta cuando evalúa la calidad de un servicio, por lo tanto, las organizaciones prestadoras de servicios pueden utilizar este conocimiento como instrumento para el control de la gestión, ya que, al conocer qué es lo más valioso para el cliente, podrá orientar sus esfuerzos a entre-

\section{Cuadro 1}

Dimensiones para evaluar la calidad del servicio.

\begin{tabular}{|c|c|}
\hline Elementos tangibles & $\begin{array}{l}\text { Apariencia de las instalaciones físicas, equipos, personal y } \\
\text { materiales de comunicación. }\end{array}$ \\
\hline Confiabilidad & $\begin{array}{l}\text { Habilidad para prestar el servicio prometido de forma fiable y } \\
\text { cuidadosa }\end{array}$ \\
\hline Capacidad de respuesta & $\begin{array}{l}\text { Disposición y voluntad de los empleados para ayudar a los clientes y } \\
\text { ofrecerles un servicio rápido. }\end{array}$ \\
\hline Seguridad & $\begin{array}{l}\text { Conocimientos y atención mostrados por el personal de contacto y } \\
\text { sus habilidades para inspirar credibilidad y confianza para el cliente. }\end{array}$ \\
\hline Empatía & $\begin{array}{l}\text { Atención individualizada que el proveedor del servicio ofrece a los } \\
\text { clientes }\end{array}$ \\
\hline
\end{tabular}

Fuente: Zeithaml et al (1992). 
gar un servicio con esas características, a fin de mejorar el nivel de calidad percibido por el cliente (Setó, 2004).

En las investigaciones de marketing de servicios se han reconocido diferentes modelos como instrumentos para medir la calidad de servicio. Los más aplicados son el SERVQUAL (calidad del servicio) y el SERVPERF (calidad de servicio percibida).

El modelo SERVQUAL fue creado por Parasuraman, Zeithaml y Berry, entre 1985 y 1988. Éste define la calidad del servicio como la diferencia entre las percepciones reales por parte de los clientes y sus propias expectativas. Es un instrumento de escala múltiple que presenta un alto nivel de fiabilidad y validez, que las empresas pueden utilizar para comprender mejor las expectativas y la percepción que tienen los clientes respecto a un servicio.

Por el contrario, el modelo SERVPERF consta de una escala centrada en la medición de la calidad percibida de los servicios, a través de la satisfacción del cliente. Consiste en un instrumento desarrollado por Cronin y Taylor en 1982, quienes probaron un modelo alternativo sustentado en el desempeño, el cual se basa en 22 de los ítems propuestos en el SERVQUAL y que sugieren mesurar la calidad sólo con el test de percepciones que propone el mismo (Valls, 2004).

Estos modelos contienen diferentes puntos de vista y han sido propuestos bajo distintos contextos socio-políticos en el mundo, sin embargo la aplicación del SERVPERF como modelo de medición de la calidad posee las siguientes ventajas:

a. No presenta a los encuestados problemas de interpretación ni redundan- cia en las preguntas planteadas, ya que se basa en la percepción del usuario.

b. El tiempo y los recursos empleados para la aplicación del instrumento se reducen debido a que este contiene la mitad de ítems que incluye el SERVQUAL, dejando espacio para incluir otras declaraciones que si aportarán información al estudio.

Bajo este modelo, la perspectiva del usuario constituye un elemento primordial tomando en cuenta que la finalidad de todo servicio es lograr la satisfacción del cliente, en cuyo caso la percepción que él tenga sobre la calidad del mismo es esencial para la su evaluación.

\section{Calidad del servicio público de aseo urbano en la ciudad de Maracaibo}

La realización de la presente investigación permitió obtener resultados tanto metodológicos, como desde el punto de vista de la aplicabilidad de las técnicas aquí descritas para medir la variable de estudio, esto con la finalidad de buscar mejoras a la sociedad y resolver las interrogantes planteadas en relación a la calidad de servicio del aseo urbano. Dichos resultados se presentan a continuación:

\subsection{Escala de medición de calidad para el servicio de aseo urbano}

Producto de la revisión bibliográfica y del análisis de los modelos teóricos más usados para medir la calidad de un servicio, se diseñó un instrumento de medición que permite valorar la calidad de servicio del aseo urbano domiciliario. 
Calidad del Servicio Público de Aseo Urbano: Caso: Municipio Maracaibo

Sáez Alejandrina; Machado Emily y Godoy Enemarsy

El instrumento está estructurado en 24 ítems distribuidos en cinco dimensiones, que definen los aspectos, que desde la perspectiva del usuario, son determinantes de la calidad del servicio. Las cinco dimensiones originales del modelo SERVPERF se definieron específicamente para el servicio de aseo urbano, quedando conceptualizadas de la siguiente manera:

- Tangibilidad: Hace referencia a la apariencia, identificación y condiciones físicas de las unidades de recolección, así como también a la apariencia del personal de recolección y del material de comunicación emitido por la institución. Aspectos que están representados en el instrumento de evaluación de la calidad de servicio del aseo urbano por los ítems 1, 2 y 3 correspondientemente.

- Confiabilidad: Habilidad para prestar el servicio prometido de forma fiable y cuidadosa, es decir, que en la práctica la institución proveedora del servicio cumpla con lo que promete a sus clientes, y que preste un buen servicio en concordancia con lo ofrecido a estos. Los indicadores para esta dimensión son: credibilidad (ítem 4), cumplimiento de responsabilidades (ítem 5 ), frecuencia (ítems 6 y 7), información oportuna (ítem 8), puntualidad (ítems 9 y 10).

- Capacidad de respuesta: Disposición y voluntad de los empleados para ayudar a los usuarios de forma rápida y oportuna, demostrando dedicación, interés y esfuerzo en la solución de los problemas planteados por los usuarios, a través de claros y eficaces canales de comunicación establecidos por la institución. Esta dimensión es medida empleando los siguientes indicadores: interés en solucionar los problemas (afirmaciones 11 y 12), disposición para colaborar con el usuario (afirmaciones 13 y 14), y tiempo de respuesta (afirmación 15).

- Seguridad: Habilidad de los trabajadores del instituto para trasmitir confianza exhibiendo un comportamiento respetuoso a los usuarios, y haciendo que estos se sientan tranquilos y no perciban riesgos durante la prestación del servicio, así como también para inspirar credibilidad a través de los conocimientos mostrados por el personal de contacto, al responder las inquietudes de los clientes en relación al servicio. Para esta dimensión se establecieron los siguientes indicadores: confianza (ítems 16, 19 y 20), conocimiento (ítem 17) y respeto (ítem 18).

- Empatía: Comprensión de la entidad que presta el servicio de las necesidades de los usuarios, e interés por interactuar con los clientes tanto para comunicarles los cambios y modificaciones del servicio, como para recibir sus peticiones y observaciones a fin de mejorar el mismo. Para la medición de esta dimensión se establecieron estos indicadores: atención (declaración 21), comprensión (declaración 22) y comunicación (declaraciones 23 y 24).

Atendiendo a las dimensiones definidas, el instrumento propuesto y utilizado para la determinación de la calidad del servicio público de aseo urbano domiciliario, se presenta en el Cuadro 2, donde se muestran los ítems contenidos en el mismo. 


\section{Cuadro 2}

Instrumento de evaluación de la calidad de servicio del aseo urbano.

\begin{tabular}{c} 
MODELO PROPUESTO \\
DIMENSIÓN 1: TANGIBILIDAD \\
\hline
\end{tabular}

1. Los vehículos recolectores de basura están en buen estado e identificadas con el logo de la empresa. 2. Los trabajadores que recogen los desechos sólidos cuentan con la vestimenta apropiada para realizar la labor que desempeñan.

3. El IMAU emite material de comunicación atractivo para el usuario informándole temas como: la reducción en la generación de desechos; tratamientos y formas de almacenamiento adecuados para la basura; medidas para la prevención de enfermedades y epidemias ocasionadas por la manipulación incorrecta de los desechos, entre otras.

\section{DIMENSIÓN 2: CONFIABILIDAD}

4. El IMAU cumple con lo que promete a los usuarios.

5. Los trabajadores que recogen los desechos sólidos realizan la recolección de forma cuidadosa, evitando el esparcimiento de desechos.

6 Existe falta de comunicación por parte de IMAU a los usuarios con respecto a la frecuencia de recolección de basura.

7. La recolección de desechos sólidos se realiza con una frecuencia diferente a la prometida.

8. El IMAU mantiene desinformado al usuario sobre los días y horarios de recolección de los desechos sólidos.

9. La recolección de desechos sólidos se realiza fuera de los días fijados por el IMAU.

10. La factura no llega regularmente cada trimestre.

\section{DIMENSIÓN 3: CAPACIDAD DE RESPUESTA}

11. El IMAU resuelve de manera oportuna los problemas, que usted plantea, con relación a la recolección de desechos sólidos.

12. El instituto tiene bien definidos canales de comunicación con el usuario para recibir reclamos. 13. Los empleados del IMAU están disponibles para atender a la comunidad cuando realiza un reclamo. 14. Los empleados del IMAU demuestran disposición a ayudar a la comunidad cuando ésta realiza un reclamo.

15. EI IMAU atiende de manera rápida las denuncias presentadas por la comunidad.

\section{MODELO PROPUESTO DIMENSIÓN 4: SEGURIDAD}

16. El comportamiento de los trabajadores que recogen los desechos sólidos le transmite desconfianza. 17. Los trabajadores que recogen los desechos sólidos carecen de conocimientos suficientes para responder sus inquietudes respecto al servicio.

18. Los trabajadores que recogen los desechos sólidos son irrespetuosos con los usuarios.

19. El personal de recolección de desechos sólidos utiliza una vestimenta sin la apropiada identificación como empleado del IMAU.

20. En ocasiones, el personal recolector de desechos sólidos le exige alguna recompensa para prestarle el servicio.

\section{DIMENSIÓN 5: EMPATÍA}

21. El servicio de recolección se realiza en horarios convenientes para los usuarios.

22. El IMAU comprende las necesidades específicas de sus usuarios en lo que respecta al servicio de aseo urbano.

23. La empresa notifica a los usuarios, en qué momento se realizaran modificaciones en los planes de recolección.

24. Los vehículos de recolección del IMAU exhiben en un lugar visible los teléfonos de atención al usuario (para la recepción de quejas y reclamos).

Fuente: Elaboración propia (2010) con base en Cronin y Taylor (1992). 
Calidad del Servicio Público de Aseo Urbano: Caso: Municipio Maracaibo Sáez Alejandrina; Machado Emily y Godoy Enemarsy

Para cada afirmación, el usuario tiene cinco apreciaciones diferentes: fuertemente en desacuerdo, muy en desacuerdo, ni en desacuerdo ni de acuerdo, muy de acuerdo o fuertemente de acuerdo, las cuales tienen asignadas puntuaciones del 1 al 5 respectivamente, para cuantificar las dimensiones expuestas.

Con la finalidad de categorizar y convertir en una lectura cualitativa la medición obtenida de la calidad de servicio del aseo urbano que permita interpretar el grado de satisfacción del usuario con el servicio, se determinaron clases con un ancho de 1.33 cada una; la clasificación de la calidad de servicio obtenida es presentada en el Cuadro 3.

\subsection{Medición de la calidad del servicio público de aseo urbano}

La calidad del servicio público de aseo urbano es medida a través de los resultados obtenidos con la aplicación del instrumento propuesto a una muestra de 314 usuarios, durante el periodo comprendido entre el 16 de marzo al 14 de abril de 2010. Los datos obtenidos se pro- cesaron en el paquete estadístico SPSS 15.0 y se realizaron los cálculos de las medias tanto por ítem como por dimensión, arrojando los siguientes resultados.

- Tangibilidad: Para valorar esta dimensión se utilizaron tres indicadores, de los cuales, el indicador apariencia de las unidades de recolección resultó ser el mejor evaluado con un nivel de calidad medio de 3.61 , resultado que refleja que una buena parte de los encuestados (63.64\%) estuvo de muy fuertemente de acuerdo a fuertemente de acuerdo con la afirmación sobre el buen estado e identificación de los vehículos recolectores.

En cuanto a los indicadores de apariencia del personal recolector y del material de información dirigido a los usuarios, éstos obtuvieron una calificación de 2.26 y 1.15 respectivamente, lo que los ubica en el rango de baja calidad. En el caso de la apariencia del personal de recolección más del $60 \%$ de los encuestados manifestó que estos trabajadores no cuentan con el equipo de protección personal que su labor amerita, la mayoría está desprovisto de guantes, mientras el uso de mascarillas es muy raro

Cuadro 3

Escala de medición de la calidad.

\begin{tabular}{|c|c|c|}
\hline Intervalo & Nivel de Calidad & Interpretación \\
\hline$[1,2.33)$ & Calidad Baja & $\begin{array}{l}\text { Las expectativas del usuario no son cumplidas, por lo } \\
\text { tanto percibe la calidad de servicio como inaceptable. }\end{array}$ \\
\hline$[2.33,3.67)$ & Calidad Media & $\begin{array}{l}\text { Las expectativas son medianamente cumplidas, por lo } \\
\text { que los procesos ameritan atención y acciones } \\
\text { correctivas que mejoren la calidad percibida por el } \\
\text { usuario. }\end{array}$ \\
\hline$[3.67,5]$ & Calidad Alta & $\begin{array}{l}\text { Las expectativas son satisfechas ó excedidas, por lo } \\
\text { tanto, los usuarios se encuentran ampliamente } \\
\text { satisfechos con el servicio prestado. }\end{array}$ \\
\hline
\end{tabular}

Fuente: Elaboración propia (2010). 
entre el personal de recolección, a esto se añade el hecho que buena parte de los recolectores visten de particular lo que denota la ausencia de un uniforme.

De acuerdo a estos resultados, el atractivo del material de comunicación emitido por la institución fue el que obtuvo menor puntuación debido a que el $92.33 \%$ de los entrevistados estuvo fuertemente en desacuerdo con dicha declaración. Al indagar sobre las razones que conducen a este resultado, los usuarios argumentaron que el ente proveedor del servicio en muy pocas ocasiones les hace llegar material informativo de ningún tipo, de lo cual se deduce que la Institución presenta debilidades en el uso efectivo de medios de comunicación impresos, más que fallar en el atractivo de los folletos.

En general, la dimensión tangibilidad obtuvo una calificación de 2.34 alcanzando ligeramente el nivel de calidad medio. En el Gráfico 1 se presentan desglosados por indicador los resultados obtenidos para esta dimensión.
- Confiabilidad: Globalmente resultó calificada con 1.77 ubicándose en el nivel de baja calidad.

Para medir esta dimensión se emplearon siete ítems que abarcan los cinco indicadores establecidos para la misma, cuyos resultados son muy similares, siendo calificados como de baja calidad, exceptuando el ítem cinco (5) que corresponde al indicador cumplimiento cuidadoso de responsabilidades, que fue calificado con 2.38 como de calidad media. Si bien este último fue mejor evaluado en comparación con el resto, durante la aplicación del instrumento los usuarios señalaron que durante la recolección si se rompen las bolsas y se esparcen residuos en la carretera, los trabajadores los dejan tirados allí, sin realizar esfuerzo alguno para corregir este evento indeseable.

Analizando individualmente los ítems, la credibilidad (ítem 4) alcanzó una puntuación de 1.98 lo que se traduce como que el usuario tiene poca fe en las promesas que el proveedor de servicio

Gráfico 1

Indicadores de tangibilidad.

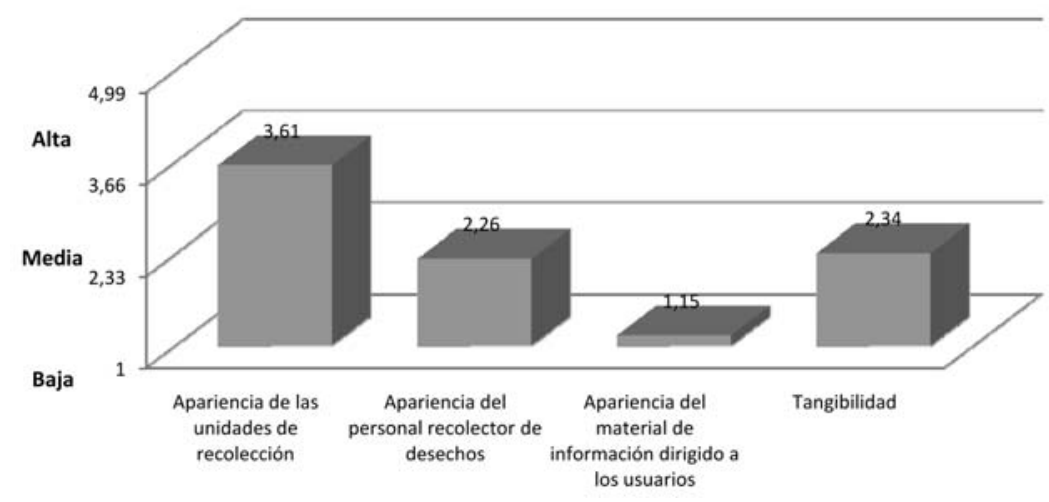

Fuente: Elaboración propia (2010). 
Calidad del Servicio Público de Aseo Urbano: Caso: Municipio Maracaibo Sáez Alejandrina; Machado Emily y Godoy Enemarsy

hace, de hecho el $70 \%$ de los ciudadanos encuestados opinan que éste no cumple con lo que promete. Igualmente el indicador frecuencia medido con los ítems 6 y 7 del instrumento fueron calificados con 1.50 y 1.80 respectivamente, debido a que el $86 \%$ de los entrevistados asegura que la institución no le comunica la periodicidad de la recolección, y casi el $80 \%$ reconoce que existe una promesa de una frecuencia de dos veces a la semana por parroquia, que no ha sido cumplida.

Con respecto al indicador información oportuna (ítem 8), fue evaluado con 1.48 resultado muy parecido al obtenido en el ítem 6 , deduciéndose de ambos una falta de comunicación entre el Instituto y el usuario. La puntualidad fue medida a través de dos ítems (9 y 10) pero enfocadas a un servicio central del aseo urbano y a uno periférico del mismo, a los que el usuario calificó con 1.78 y 1.45 respectivamente. En el caso de la puntualidad en la recolección, aproximadamente el $79 \%$ de los encuestados estuvo en desacuerdo con la afirmación; mientras que en el caso del servicio periférico de facturación el $87 \%$ coincide que la factura no llega trimestralmente y expresan que les gustaría recibir con regularidad la factura.

En el Gráfico 2 se presentan los resultados obtenidos para cada indicador de esta dimensión.

- Capacidad de Respuesta: Esta dimensión ha sido calificada con 1.70 en un nivel de calidad bajo, lo que quiere decir que la Institución tiene muy poca capacidad de respuesta ante las necesidades y exigencias de la comunidad servida. Para el indicador interés en solucionar los problemas, medido por los ítems 11 y 12, que fueron evaluados con 1.63 y 1.76 cada uno; cerca del $83 \%$ está en desacuerdo con la afirmación de que el proveedor de servicio resuelve de manera oportuna los

Gráfico 2

Indicadores de confiabilidad.

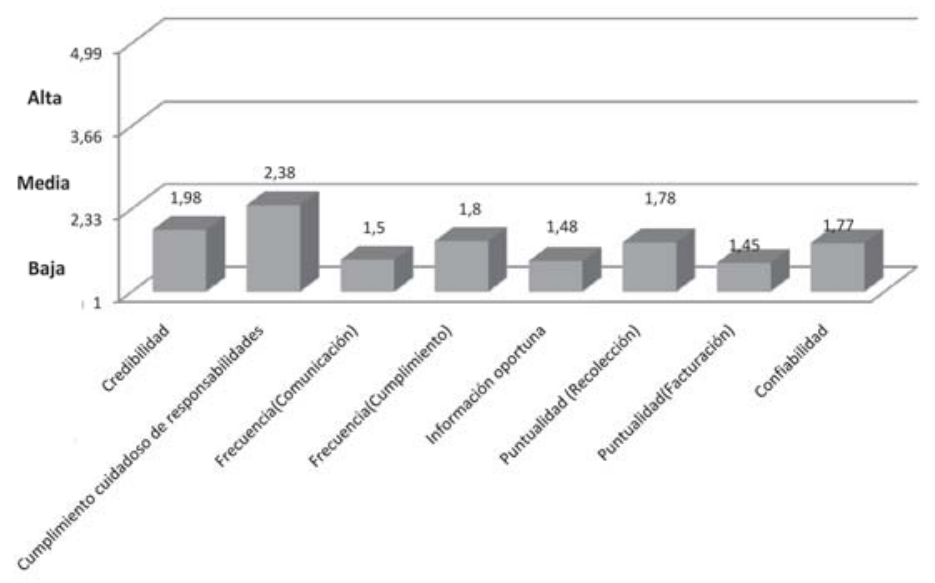

Fuente: Elaboración propia (2010). 
problemas que se plantean en torno a la recolección de desechos, y sólo el $14.42 \%$ opina que el ente operador del servicio tiene bien definidos los canales de comunicación para recibir reclamos. El indicador disposición para colaborar con los usuarios, calificado con 1.73, permite inferir poca receptividad por parte del proveedor de servicio para con los reclamos. De igual manera alrededor del $76 \%$ de los entrevistados coincide en la falta de voluntad y el escaso esfuerzo mostrado por la institución para solucionar los problemas de la comunidad en relación al servicio, que se evidencia en la calificación obtenida de 1.79 para la declaración 14.

Con respecto al indicador tiempo de respuesta, éste obtuvo una puntuación de 1.60 , la más baja de toda la dimensión, debido a que el $83 \%$ de los usuarios opina que el Instituto no atiende con la suficiente rapidez las denuncias presentadas por la comunidad.
En el Gráfico 3 se presentan los resultados obtenidos para los indicadores correspondientes a esta dimensión.

- Seguridad: La dimensión seguridad en términos generales, es percibida por los usuarios como de calidad media siendo evaluada con 3.13 que la ubica en este renglón. Para llegar a este resultado se consideraron tres indicadores fundamentales: confianza, conocimiento y respeto. El indicador confianza se evaluó por medio de tres ítems (16, 19 y 20) cuyos resultados alcanzaron una puntuación promedio de $3.16,2.40$ y 3.21 respectivamente y que abarcan aspectos como el comportamiento del personal de recolección, identificación del mismo, y la seguridad en las transacciones al momento de proveer el servicio.

Con respecto a la confianza en las transacciones (ítem 20), este se enfocó hacia el aprovechamiento y adquisición de beneficios adicionales por parte del personal de recolección, provenientes de la exigencia de recompensas a cambio

\section{Gráfico 3 \\ Indicadores de capacidad de respuesta.}

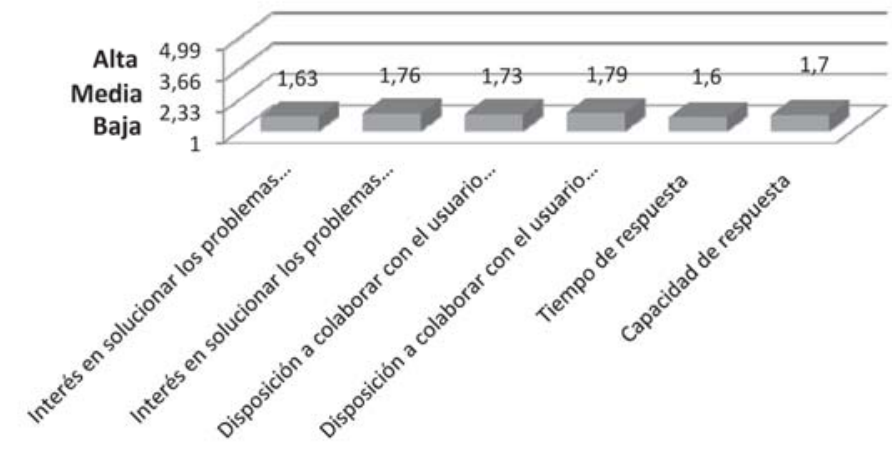

Fuente: Elaboración propia (2010). 
Calidad del Servicio Público de Aseo Urbano: Caso: Municipio Maracaibo Sáez Alejandrina; Machado Emily y Godoy Enemarsy

de la prestación del servicio. Según el resultado arrojado para este, el $49.20 \%$ de los usuarios señaló que en ocasiones los trabajadores piden un pago para llevarse escombros, ramas, y otros desechos de jardinería. El indicador mejor evaluado para esta dimensión e incluso para el total de atributos considerados en la medición, es el respeto, calificado con 4.19 ubicándose en la categoría de calidad alta, ya que el $79.93 \%$ de los usuarios expresa ser tratado con decoro y educación.

En el Gráfico 4 se presentan los resultados obtenidos para los indicadores de esta dimensión.

- Empatía: Definida como la atención individualizada al usuario, se evalúa por la atención (ítem 21), que para efectos del servicio de aseo urbano está relacionada con los horarios de recolección, resultando calificada por los usuarios con 2.77 lo que la posiciona dentro del rango de calidad media, debido a que el $49.68 \%$ de los encuestados opina que los horarios establecidos para la reco- lección no les son convenientes, sobre todo los nocturnos.

La comprensión (ítem 22), un segundo indicador de esta dimensión, obtuvo una puntuación de 2.23 que según la escala establecida es indicio de baja calidad. Esto es consecuencia de la opinión que tiene el usuario acerca de cuanto son comprendidas sus necesidades. En este sentido, el $66.56 \%$ percibe que sus necesidades no son entendidas, alegando no sólo el hecho de no estar totalmente de acuerdo con los horarios, sino también todas las irregularidades cometidas durante la prestación del servicio.

En cuanto al indicador comunicación, este fue evaluado según dos aspectos: la comunicación con los usuarios y los mecanismos de comunicación. Con relación al primer aspecto, sobre si la empresa comunica a los usuarios en que momento se realizarán modificaciones en los planes de recolección (ítem 23), el $95.21 \%$ de los encuestados estuvo en desacuerdo con esta afirmación. Además, el usuario percibe que el ente operador

Gráfico 4

Indicadores de seguridad.

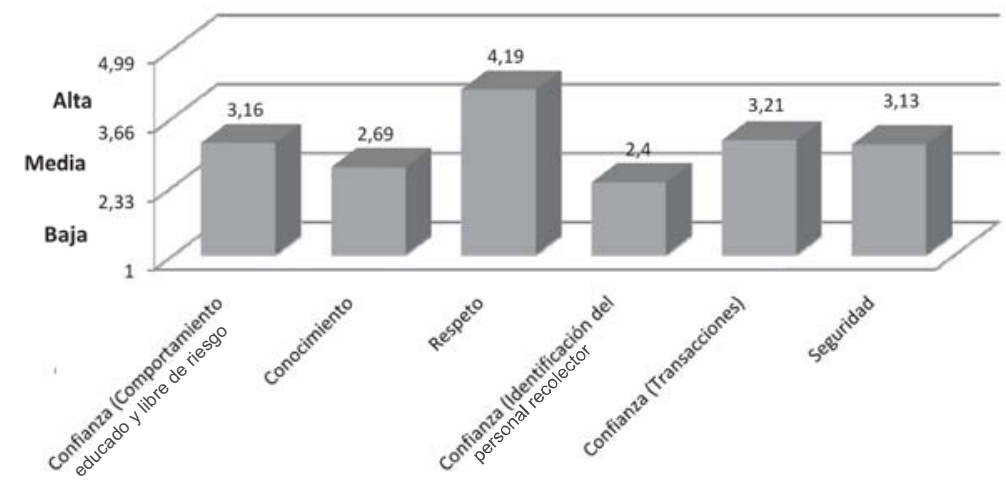

Fuente: Elaboración propia (2010). 
no le informa lo suficiente en relación al servicio, de allí que este ítem obtuviera la calificación más baja para la dimensión empatía con un puntaje de 1.19.

El segundo aspecto del indicador comunicación, está vinculado a los mecanismos de los cuales dispone el Instituto para recibir las quejas, como lo son los números telefónicos, pero tener un mecanismo no es suficiente, es necesario dar a conocer a los usuarios los medios con que se cuenta, lo que indicaría que realmente la institución tiene interés en escuchar a sus clientes y en conocer sus demandas, contribuyendo a tener una relación empática entre ambos actores. El ítem 24 buscaba verificar si realmente las unidades exhibían en un lugar visible los números de atención al público. Ante esta declaración los entrevistados estuvieron de acuerdo en un $34.22 \%$ en decir que efectivamente las unidades muestran dichos teléfonos, de allí que la calificación obtenida por este aspecto sea de 2.64.

En términos generales, la dimensión empatía alcanzó una puntuación de
2.21, según la cual, es calificada como de baja calidad.

En el Gráfico 5 se muestran los resultados obtenidos para los indicadores de esta dimensión.

Una vez analizados individualmente los indicadores y las dimensiones, se compararon las calificaciones obtenidas para cada una de ellas, a fin de identificar aquellas percibidas por el usuario como de menor calidad y que requieren de un mayor esfuerzo por el Instituto para mejorar significativamente, de manera que se genere valor al servicio y satisfacción al usuario. En el Gráfico 6 se presentan los resultados para las cinco dimensiones utilizadas para evaluar la calidad de servicio del aseo urbano desde la percepción del usuario.

De las cinco dimensiones estudiadas, la confiabilidad, capacidad de respuesta y empatía fueron percibidas como de baja calidad, mientras que la tangibilidad y seguridad, fueron evaluadas como de calidad media.

Ninguna fue calificada como calidad alta, y la que es mejor percibida por

Gráfico 5

Indicadores de empatía.

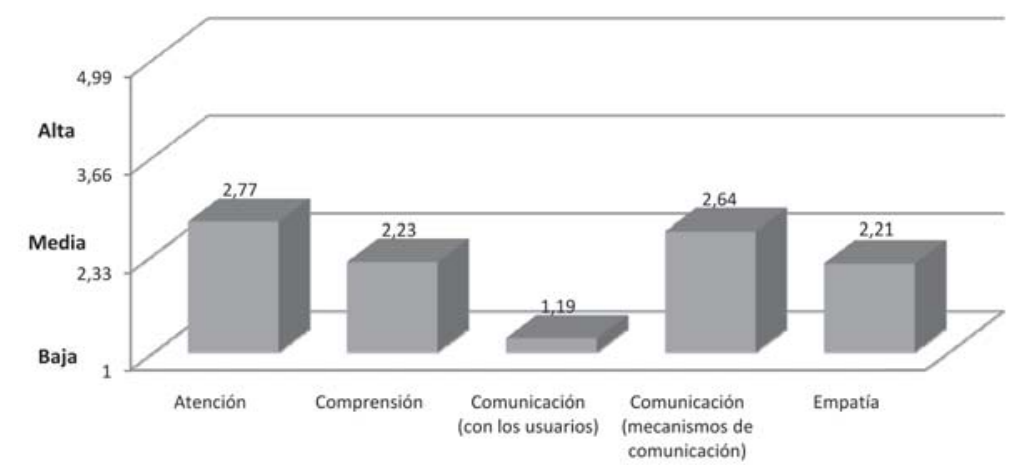

Fuente: Elaboración propia (2010). 
Calidad del Servicio Público de Aseo Urbano: Caso: Municipio Maracaibo Sáez Alejandrina; Machado Emily y Godoy Enemarsy

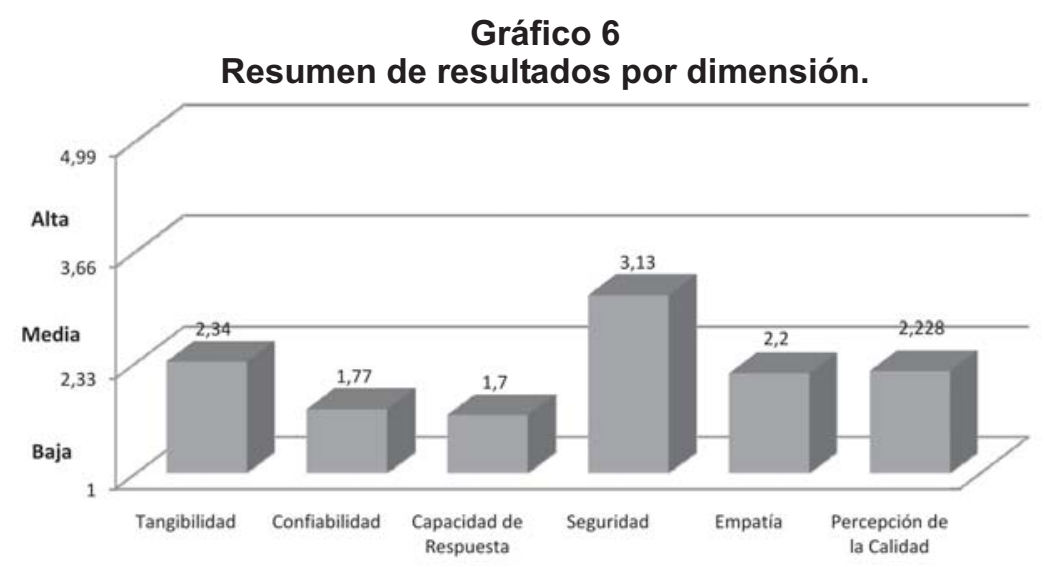

Fuente: Elaboración propia (2010).

\section{Gráfico 7}

Dimensiones y percepción de la calidad por parroquias.

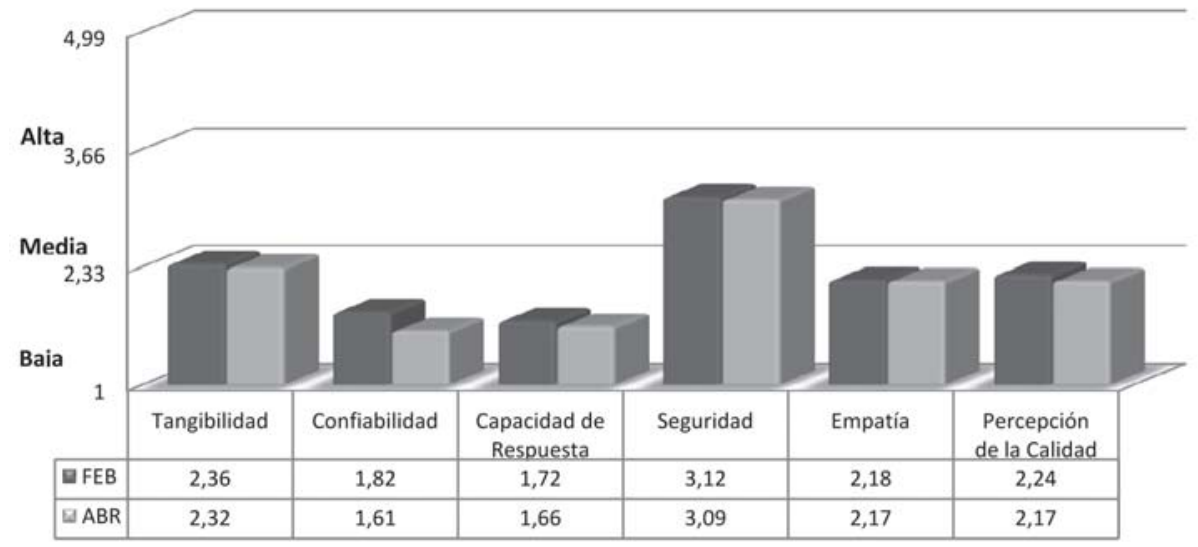

Fuente: Elaboración propia (2010).

los usuarios pese a las situaciones indeseables identificadas es la seguridad. La percepción promedio de todas las dimensiones para la calidad resultó ser de 2.228 por lo que desde el punto de vista del usuario, el servicio de aseo urbano, califica en la categoría de baja calidad, siendo ésta inaceptable para los ciudada- nos, quienes se sienten insatisfechos con el servicio recibido.

En el Gráfico 7 se presentan los resultados para cada parroquia por dimensión y la percepción de la calidad. La similitud en los resultados se atribuye a que son parroquias que reciben el servicio por la misma Institución. 


\section{Gráfico 8}

\section{Resumen de resultados por dimensión y atributos.}

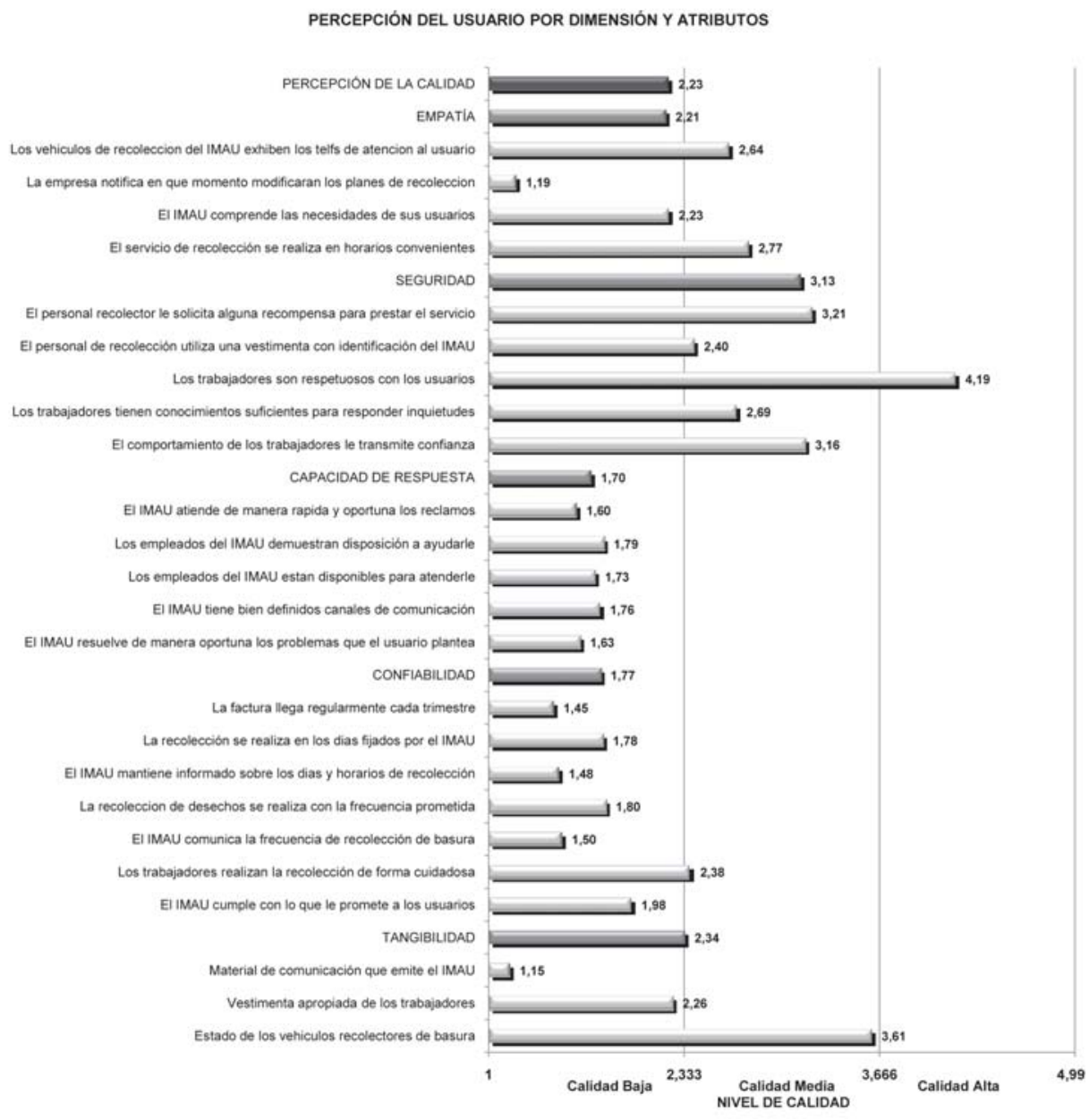

Fuente: Elaboración propia (2010).

En el Gráfico 8 se presentan los resultados para la percepción del usuario y para las dimensiones con sus respectivos atributos.

\section{Conclusiones}

La baja calificación de la calidad de servicio del aseo urbano percibida por los usuarios indica que la ciudadanía está re- 
Calidad del Servicio Público de Aseo Urbano: Caso: Municipio Maracaibo

Sáez Alejandrina; Machado Emily y Godoy Enemarsy

cibiendo un servicio que considera inaceptable desde el punto de vista ambiental, estético y sanitario; resultado que indica que el servicio prestado dista de cubrir las expectativas mínimas de la población y que está muy lejos de contribuir con el incremento de su calidad de vida.

Del análisis de las dimensiones consideradas a efectos de la investigación se señalan como factores claves del servicio de aseo urbano la capacidad que tiene el ente prestador del mismo para dar respuesta a las necesidades de los usuarios, así como el cumplimiento de la frecuencia y horario de recolección de desechos establecido. Es importante señalar que los usuarios tienen una limitada visión del servicio, la cual se reduce a la recolección, y su satisfacción está condicionada en gran medida al cumplimiento de la frecuencia, la cual se convierte en un aspecto crítico del servicio de aseo urbano.

Este resultado señala que el prestador del servicio debe orientar sus esfuerzos a mejorar su credibilidad, y sobre todo a ser efectivo en la entrega de un servicio puntual acorde a las expectativas del usuario.

Así mismo, con el objeto de incrementar la calidad del servicio, la Institución debe asegurarse de comunicar efectivamente a los usuarios las frecuencias de recolección, y la oportuna realización de operativos especiales de limpieza, lo que a su vez amerita ampliar y fortalecer los mecanismos de comunicación con el usuario para la recepción de reclamos y quejas, siendo enfáticamente importante revisar los procedimientos para el tratamiento y seguimiento de las denuncias realizadas por los usuarios y los tiempos de respuesta a las mismas, los cuales deben ser reducidos.

Conjuntamente, para cumplir con los aspectos citados, debe garantizarse el adecuado funcionamiento de las unidades recolectoras así como mejorar el aspecto físico de las mismas, dotar regularmente al personal de recolección del apropiado equipo de protección personal, uniformar al personal de recolección y asegurarse que los recolectores conozcan las formas adecuadas para manipular los contenedores y recipientes de almacenamiento de los desechos.

Finalmente, se infiere del estudio que existe poca comprensión de las necesidades y expectativas de los usuarios por parte del instituto que presta el servicio de aseo urbano, el cual debe orientar sus esfuerzos al establecimiento de estrategias que permitan incrementar la interacción entre las partes, con la finalidad de obtener la retroalimentación que requiere del usuario y diseñar el servicio acorde a las perspectivas del mismo.

\section{Referencias Bibliográficas}

Acurio, Guido; Rossin, Antonio; Texeira, Paulo; Cepeda, Francisco (1998). Diagnostico de la situación del manejo de residuos sólidos municipales en América Latina y el Caribe. Organización Panamericana de la Salud. Disponible en: http://www.iadb.org/sds/doc/ENV107ARossinE.pdf.Fecha de consulta: 11 de Octubre de 2009.

Asamblea Nacional de la República Bolivariana de Venezuela (2005). Ley Orgánica del poder Público Municipal. Gaceta Oficial $N^{\circ} 38.204$. Caracas, Venezuela. 
Asamblea Nacional de la República Bolivariana de Venezuela (2006). Proyecto de Ley orgánica de los servicios públicos.

Cronin, J. y Tailor, S. (1992). Measuring service quality: a reexamination and extension. Journal of Marketing. Vol. 56, pp. 55-68.

Pride, William M.; Ferrel, O.C.; Rosas Lopetegui, Gloria E. (1997). Marketing: Conceptos y estrategias. México. Editorial McGraw-Hill.

Setó P., Dolors (2004). De la Calidad de servicio a la fidelidad del cliente, España, Editorial ESIC.
Stanton, William; Etzel, Michael; Walter, Bruce (2004). Fundamentos de marketing, México, Editorial McGraw-Hill.

Valls F., Wilfredo (2004). Modelo RESORTQUAL para la evaluación de la calidad percibida del servicio en un destino turístico de sol y playa. Servicio al cliente. Disponible en: http://www.gestiopolis.com/recursos/documentos/fulldocs/mar1/modresortqual.htm Fecha de consulta: 08 de Febrero de 2009.

Zeithaml, Valarie; Parasuraman, A.; Berry, Leonard (1992). Calidad total en la Gestión de Servicio. España. Editorial Díaz de Santos. 